\title{
Körperliche Aktivität und Sport bei Typ-2-Diabetes
}

\section{Physical Activity and Sports in Patients With Type 2 Diabetes}

Andreas M. Nieß, Ansgar Thiel

\begin{abstract}
Körperliche Aktivität ist ein überaus wichtiger Bestandteil der Therapie und Prävention des Typ-2-Diabetes - leider scheitern wirksame Trainingsprogramme häufig an mangelnder Motivation der betroffenen Patienten. Dieser Beitrag beleuchtet die positiven Effekte des körperlichen Trainings, erläutert die Leitlinienempfehlungen und gibt praktische Tipps zur individuellen Trainingsplanung und -beratung.
\end{abstract}

\section{Einleitung}

Ein ausreichender Umfang an körperlicher Aktivität zählt zu den wichtigsten nicht medikamentösen Maßnahmen zur Prävention [1] und Therapie des Typ-2-Diabetes [2]. Dabei verbessert ein regelmäßiges körperliches Training nicht nur die glykämische Kontrolle [3]. Es senkt auch das kardiovaskuläre Risiko [4, 5], wirkt unterstützend bei der Reduktion von Übergewicht, verbessert und stabilisiert die körperliche Fitness und Mobilität [6, 7]. Somit senkt ein ausreichender Umfang an körperlichem Training bei Patienten mit Typ-2-Diabetes sowohl die Gesamtmortalität als auch die kardiovaskuläre Sterblichkeit [8].

Allerdings erreicht weltweit nur ein kleinerer Teil der Menschen den unter einer präventiven Zielsetzung empfohlenen Mindestumfang an körperlicher Aktivität [8]. Bei Menschen mit Diabetes liegt der Anteil derer, die regelmäßig und ausreichend körperlich aktiv sind, bei lediglich knapp einem Drittel [9]. Hinzu kommt, dass bei einem großen Bevölkerungsanteil nicht nur der Gesamtumfang an körperlicher Aktivität zu gering bemessen ist. Auf der anderen Seite haben auch die Zeiten ausschließlicher Inaktivität, insbesondere verursacht durch längeres Sitzen, zugenommen. Dabei zeigt sich in den vorliegenden epidemiologischen Studien ein positiver Zusammenhang zwischen der täglichen Sitzdauer und dem Krankheitsrisiko [10] - auch für einen Typ-2-Diabetes [11]. Körperliche Aktivität und Training weisen also wie bei kaum einer anderen Entität ein hohes und breites Potenzial der Wirksamkeit auf - dennoch bleiben sie letztendlich als präventives und therapeutisches Instrument häufig ungenutzt.

\section{Merke}

Den Ärzten, die Patienten mit Typ-2-Diabetes behandeln, kommt eine Schlüsselrolle bei der Beratung und Motivation zu einem körperlichen Training und aktiven Lebensstil zu.

\section{Wirksamkeit körperlichen Trainings}

\section{Effekte auf die glykämische Stoffwechsellage}

Mithilfe eines regelmäßigen körperlichen Trainings kann beim Patienten mit Typ-2-Diabetes direkt auf die zugrunde liegende Pathophysiologie einer gestörten Glukoseregulation Einfluss genommen werden. So konnte eine Metaanalyse mit insgesamt 8538 eingeschlossenen Patienten [2] gut dokumentieren: Unter einem strukturierten, begleiteten Ausdauer-, Kraft- oder einem kombinierten Training von mindestens 12 Wochen Dauer lässt sich die glykämische Stoffwechsellage in klinisch relevantem Ausmaß verbessern. Beim aeroben Ausdauertraining reduzierte sich der HbA1c-Wert um durchschnittlich 0,73\%, beim Kraftausdauertraining um $0,57 \%$ und beim kombinierten Training um $0,51 \%$. Lag der wöchentliche Trainingsumfang oberhalb von 2,5 Stunden, so war sogar eine Abnahme des HbA1c um 0,89\% im Mittel zu verzeichnen.

Neueren Daten zufolge können beim dynamischen Ausdauertraining möglicherweise höhere Belastungsintensitäten bei gleichzeitig kürzerer Belastungsdauer den günstigen Effekt auf die glykämische Stoffwechsellage noch steigern [12]. Zwar ist die Studienlage hierzu noch nicht so umfangreich wie für das moderat-intensive Ausdauertraining, doch ein Review zeigt: Bei einem Teil der Studien verbesserte sich die Insulinsensitivität unter einem höher-intensiven Intervalltraining (HIT) deutlicher als unter einem moderat-intensiven Training nach der Dauermethode [13].

\section{HINTERGRUND}

Mittlerweile konnten Mechanismen identifiziert werden, die den günstigen Effekt körperlichen Trainings auf die glykämische Stoffwechsellage erklären. So kommt es unter wiederholten Muskelkontrak- 
tionen sowohl zu einer gesteigerten myozellulären Expression des Glukosetransporters-4 (GLUT-4) als auch zu einer Translokation des Proteins zur Zellmembran [14]. Als das diesen Effekt vermittelnde Signalmolekül konnte die Adenosin-Monophosphat(AMP-)aktivierte Kinase (AMPK) identifiziert werden, welche durch die kontraktionsinduzierte Zunahme von AMP stimuliert wird [15]. Des Weiteren werden im arbeitenden Skelettmuskel Transkriptionsfaktoren wie die Phosphoinositid-3-Kinase (PI3K) sowie die Mitogen-activated-Protein-(MAP-)Kinasen aktiviert. Dies hat einen günstigen Einfluss auf die Insulinwirkung und erhöht die zelluläre Glukoseaufnahme [16].

\section{Fettsäureoxidation und ektope Fettspeicher}

Im Rahmen der mitochondrialen Biogenese und der damit einhergehenden Zunahme der mitochondrialen Enzymkapazität verbessert sich unter einem Ausdauertraining die oxidative Energiebereitstellung. Dieser Prozess wird von einer Aktivitätszunahme membranöser und zellulärer Fettsäuretransporter begleitet und begünstigt die Einschleusung plasmatischer Fettsäuren und myozellulärer Triglyzeride in das Mitochondrium [17]. Dieser Trainingseffekt wirkt der beim Patienten mit Typ-2-Diabetes schon frühzeitig nachweisbaren ektopen Lipidakkumulation im Muskel entgegen. Er senkt die Konzentration eingelagerter Triglyzeride und verringert gleichzeitig die Insulinresistenz [17].

Noch deutlicher ausgeprägt sind die Effekte eines körperlichen Trainings auf die Verringerung intrahepatischer Triglyzeride, die in der Mehrzahl der vorliegenden Studien nachgewiesen werden konnte $[18,19]$. Ein Ausdauertraining hat dabei eine stärkere Wirkung als Krafttraining [20], jedoch sind auch bei Letzterem Effekte nachweisbar [19]. Dies ist bedeutsam, da eine hepatische Lipidakkumulation eine wichtige Rolle bei der Entstehung des Typ2-Diabetes und kardiovaskulärer Erkrankungen spielt. Günstige Auswirkungen hat ein körperliches Training schließlich auch auf Cholesterin und Triglyzeride inklusive dem Profil der Lipoprotein-Subklassen [21] - auch davon profitieren Patienten mit Typ-2-Diabetes [22].

\section{Merke}

Ausdauer-, Krafttraining oder ein kombiniertes Training verbessert bei Patienten mit Typ-2-Diabetes die glykämische Stoffwechsellage und zeigt positive Effekte auf Triglyzeride, Cholesterin und das Profil der Lipoprotein-Subklassen.

\section{Gewichtsreduktion und -kontrolle}

Die Reduktion eines erhöhten Körpergewichts stellt beim Typ-2-Diabetes eine wichtige Maßnahme der Lebensstilintervention dar [23]. Neben diätetischen Maßnahmen ist die Steigerung des Energieumsatzes durch regelmäßige körperliche Aktivität als zentrales Instrument der Gewichtsreduktion anzusehen. Um eine signifikante und nachhaltige Gewichtsreduktion zu realisieren, ist - im Vergleich zu anderen trainingsinduzierten Effekten - in der Regel jedoch ein höherer Aktivitätsumfang notwendig. So wird für eine moderate Gewichtsreduktion von 3 \% ein zusätzlicher Energieverbrauch in einer Größenordnung von $1200-2000 \mathrm{kcal}$ pro Woche als notwendig erachtet. Dies entspricht einem Volumen von wöchentlich 150 - 250 min an moderat-intensiven Ausdauerbelastungen [23, 24]. Wird eine raschere Gewichtsabnahme angestrebt, muss der dafür notwendige Umfang an ausdauerbasierten Belastungen mit 250-300 min pro Woche noch höher bemessen werden [23]. Ein ausschließliches Krafttraining mit dem Ziel einer Gewichtsreduktion wird als nicht ausreichend wirksam angesehen [23], da es zu einer geringeren Steigerung des Energieverbrauchs führt [20]. Es ist allerdings begleitend sinnvoll, um durch den dadurch erreichten Muskelaufbau die Belastbarkeit für ein dynamisches Ausdauertraining zu steigern [24].

\section{Antiinflammatorische Effekte}

Proinflammatorischen Prozessen wird bei Entstehung und dem Progress eines Typ-2-Diabetes eine wichtige Rolle zugeschrieben [25]. Körperliche Aktivität besitzt eine antiinflammatorische Wirkung, die sowohl direkt als auch indirekt durch wiederholte Muskelaktivität vermittelt wird. Als direkter Effekt wird eine belastungsinduzierte Expressionszunahme des Myokins Interleukin-6 (IL-6) im arbeitenden Skelettmuskel beschrieben. Diese führt zu einer Inhibition des Signalings von Tumornekrosefaktor- $\alpha$ (TNF- $\alpha)$ und des Interleukin-1 $\beta$ (IL-1 $\beta)$. Beide proinflammatorische Zytokine haben eine wichtige pathophysiologische Bedeutung bei der Entwicklung der Insulinresistenz (TNF- $\alpha$ ) und des Betazellversagens (IL-1 $\beta$ ) [26]. Neben diesen direkten Wirkungen entfalten sich durch die aktivitätsinduzierte Abnahme der ektopen Lipidakkumulation ebenfalls antientzündliche Effekte [27]. Diese Wirkungen körperlicher Aktivität bei Patienten mit Typ-2-Diabetes konnten mittlerweile in einer Reihe von Studien belegt werden. Dabei ließ sich insbesondere eine Abnahme der Konzentrationen des C-reaktiven Proteins (CRP) und der Zytokine TNF- $\alpha$ und IL-1 $\beta$ aufzeigen $[28,29]$.

\section{Kardiovaskuläre Effekte}

Das beim Typ-2-Diabetes erhöhte kardiovaskuläre Risiko kann durch ein körperliches Trainingsprogramm verringert werden [4]. In einer multifaktoriellen Interventionsstudie konnte gezeigt werden: Die trainingsinduzierten günstigen Effekte auf die glykämische Stoffwechsellage, das LDL-Cholesterin und auf inflammatorische Marker werden auch von einer Verbesserung der koronaren Endothelfunktion begleitet [30]. Körperliches Training führt dabei nicht 
nur zu einer partiellen Korrektur der endothelialen Dysfunktion, sondern damit vergesellschaftet nehmen auch inflammatorische Prozesse in der Gefäßwand ab [31].

Diese Anpassungsprozesse wirken auch auf das mikrovaskuläre Gefäßbett und verringern die beim Typ-2-Diabetes zu beobachtende Gefäßrarefizierung im Skelettmuskel [32]. Zudem bildet sich der Trainingseffekt auch in einer Abnahme der systolischen und diastolischen Blutdruckwerte ab [22]. Am Herzen selbst kann ein körperliches Training zu einer Verringerung der kardialen autonomen Dysfunktion beitragen [33]. Diese stellt einen nicht zu unterschätzenden Risikofaktor für stumme Myokardischämien und kardiovaskuläre Ereignisse dar [34].

Merke

Körperliches Training bei Typ-2-Diabetes hat eine antiinflammatorische Wirkung, verbessert die koronare Endothelfunktion und verringert die kardiale autonome Dysfunktion.

\section{Körperliche Fitness}

Ein weiteres Ziel des Bewegungstrainings bei Patienten mit Typ-2-Diabetes umfasst eine Stabilisierung oder Verbesserung der körperlichen Fitness. In diesem Kontext umschreibt „Fitness“ in erster Linie die aerobe oder kardiorespiratorische Fitness, die mittels einer spiroergometrischen Bestimmung der maximalen Sauerstoffaufnahme $\left(\mathrm{VO}_{2 \max }\right)$ ermittelt werden kann. Eine höhere $\mathrm{VO}_{2 \max }$ korreliert auch bei Patienten mit Typ-2-Diabetes negativ mit dem Sterblichkeitsrisiko [35].

Interessanterweise zeigt die körperliche Fitness einen engeren Zusammenhang mit dem Sterblichkeitsrisiko als ein erhöhter Body Mass Index (BMI). In einer longitudinal angelegten Beobachtungsstudie fanden Lee et al. [36]: Im Falle einer Abnahme der körperlichen Fitness über 6 Jahre erhöht sich trotz unveränderten Körpergewichts das Sterblichkeitsrisiko um den Faktor 1,77. Umgekehrt lässt eine Zunahme des BMI bei unveränderter Fitness das Mortalitätsrisiko jedoch nur mit einer Hazard Ratio von 1,29 ansteigen. Gut nachgewiesen ist mittlerweile:

- Eine Zunahme der körperlichen Fitness geht mit einem abnehmenden Risiko sowohl für ein metabolisches Syndrom als auch für einen Typ-2-Diabetes einher und

- bei Patienten mit Typ-2-Diabetes verbessert eine Steigerung der Fitness deutlich die glykämische Stoffwechsellage [37].

Allerdings weist die körperliche Fitness eine genetische Determinierung auf. So zeigte sich: Die Erhöhung des $\mathrm{VO}_{2 \text { max }}$ durch körperliches Training fällt bei gesunden Verwandten von Patienten mit Typ-2-Diabetes geringer aus als bei Personen ohne positive Familienanamnese selbst nach Adjustierung für den Aktivitätsumfang [38].

\section{Individuelle Trainingsantwort}

Ein interessanter und klinisch relevanter Aspekt ist, dass die Antwort auf ein regelmäßiges körperliches Training eine deutliche individuelle Variabilität aufweist. Im Rahmen der HERITAGE-Studie wurden bei 720 Gesunden individuelle Veränderungen der maximalen Sauerstoffaufnahme infolge von körperlichem Training von $-50 \mathrm{ml}$ bis $+1100 \mathrm{ml}$ beobachtet [39].

In weiteren Trainingsstudien wurde diese Beobachtung auch beim Trainingseffekt auf kardiometabole Risikofaktoren wie Blutdruck, Triglyzeride und Nüchterninsulin dokumentiert. Zwischen 10 und $20 \%$ der Untersuchten profitierten dabei nicht von einer aktivitätsbasierten Intervention [40]. Hinsichtlich der Effekte auf die Glukosehomöostase liegt der Anteil der Nonresponder je nach Interventionsform teilweise noch höher [41]. Allerdings zeigte sich in der mit Patienten mit Typ-2-Diabetes durchgeführten HART-D-Studie teilweise nur eine geringe Zunahme der kardiorespiratorischen Fitness - dennoch verbesserten sich bei allen Teilnehmern ihre metabolischen Zielvariablen [42].

Das Nichtansprechen einer Zielgröße bedeutet also nicht, dass andere relevante Endpunkte unter körperlichem Training ebenfalls keine Anpassung zeigen. Beispielsweise lag auch in einer zusammenführenden Analyse mehrerer Interventionsstudien der Anteil der Nonresponder für eine Zielgröße isoliert betrachtet bei 31 \%. Bei gleichzeitiger Betrachtung dreier Endpunkte betrug er jedoch nur noch knapp $1 \%$ [40]. Daraus lässt sich folgern: Auch bei Patienten, die primär kein Ansprechen auf ein körperliches Training zeigen, ist eine aktivitätsbasierte Lebensstilintervention geboten.

Merke

Das Ansprechen auf ein körperliches Trainingsprogramm ist individuell sehr unterschiedlich. Die Verbesserung der metabolischen Werte ist auch unabhängig einer Zunahme der kardiorespiratorischen Fitness möglich.

\section{ZUSATZINFO}

\section{Pädiktive Faktoren}

Beim Nichtansprechen auf körperliche Aktivität scheint die Form des praktizierten Trainingsprogramms eine wichtige Rolle zu spielen. Aus diesem Grund wäre es hilfreich, Nonresponder bereits im Vorhinein identifizieren zu können. Damit wäre es möglich, früher verstärkt auf andere therapeutische Optionen zurückzugreifen und ggf. ein modifiziertes Trainingsprogramm durchzuführen. Im Tübinger Lebensstil Interventionsprogramm (TULIP) waren folgende Faktoren mit einem schlechteren Ansprechen des Glukosestoffwechsels assoziiert [41]: 
- geringe Insulinsensitivität

- geringe kardiorespiratorische Fitness

- höheres viszerales und hepatisches Fett

- höhere Plasmakonzentrationen von Fetuin A

Inwieweit solche Variablen in Zukunft in der klinischen Praxis zur individualisierten Therapie genutzt werden können, muss derzeit jedoch noch offenbleiben.

\section{Körperliche Inaktivität und sedenter Lebensstil}

Seit mehreren Jahren wird in verstärktem Maße das Sitzen als möglicher Risikofaktor für kardiometabolische Erkrankungen diskutiert. In einer Metaanalyse [11] ergibt sich für Personen, die ihren Alltag vorwiegend sitzend verbringen, ein relatives Risiko von

- 1,22 für die Gesamtsterblichkeit,

- 1,15 für die kardiovaskuläre Sterblichkeit und

- 1,91 für das Auftreten eines Typ-2-Diabetes.

Allerdings wird derzeit kontrovers diskutiert, ob regelmäßige längere Sitzphasen einen eigenständigen Risikofaktor darstellen, der sich unabhängig vom Gesamtumfang an Alltags-, Freizeit- und Sportaktivitäten ungünstig auswirkt.

Durch Unterbrechung längerer Phasen der Inaktivität kann die glykämische Stoffwechsellage günstig beeinflusst werden. Diskutiert wird dabei u. a., ob reine Stehpausen ausreichen oder ob Pausen mit körperlicher Aktivität zu verbinden sind. Henson et al. [43] beobachteten bei postmenopausalen Frauen sowohl für reine Steh- als auch kurze Gehpausen günstige Effekte auf die postprandiale metabolische Antwort. Dagegen wirkten sich in einer anderen Studie [44] ausschließliche Stehpausen nicht auf das postprandiale Verhalten der Plasmaglukose aus.

Ungeklärt ist auch, ob mehrere kürzere Aktivitätspausen einer einzelnen längeren Aktivitätsepisode hinsichtlich der Induktion günstiger kardiometaboler Effekte überlegen sein könnten [45]. Immerhin führte bei Patienten mit Typ-2-Diabetes eine einzelne Ausdauerbelastung über 45 min zu einem günstigeren Blutzuckertagesprofil als 3 über den Tag verteilte 15-minütige Kurzbelastungen gleicher moderat-intensiver Intensität [46].

\section{Was empfehlen die Leitlinien?}

Seit der erstmaligen Empfehlung körperlicher Aktivität in den Positionspapieren zur Prävention und Therapie des Typ-2-Diabetes haben sich die Leitlinien zum Training bei Typ-2-Diabetes kontinuierlich weiterentwickelt [34, 47]. Auf der Basis der nunmehr umfangreicheren Studienlage werden mittlerweile 3 Ansätze zur körperlichen Aktivität empfohlen ( $\vee$ Tab. 1).

- Tab. 1 Empfehlungen der American Diabetes Association (ADA) zum körperlichen Training [34].

\section{Empfehlung}

Evidenzgrad

Erwachsene mit Diabetes sollen angehalten werden, mindestens 150 min pro Woche an moderat-intensiven aeroben Ausdauerbelastungen ( 50 - $70 \%$ der maximalen Herzfrequenz) zu absolvieren. Diese sollten auf mindestens 3 Tage pro Woche verteilt sein und es sollten jeweils nicht mehr als 2 trainingsfreie Tage dazwischen liegen.

Alle Personen - auch Diabetes-Patienten - sollten längere Inaktivitätsphasen verringern und Sitzphasen mindestens alle 90 min unterbrechen.

Sofern keine Kontraindikationen bestehen, wird Erwachsenen mit Typ-2-Diabetes ein Krafttraining mit zumindest zweimaliger Durchführung pro Woche empfohlen.

A

\section{B}

A

Die Umsetzung der Aktivitäts- und Trainingsempfehlungen ist also mithilfe verschiedener, auch kombinierbarer Konzepte möglich. Zudem wird empfohlen, neben einem strukturierten Trainingsprogramm eine Steigerung des Umfangs an körperlicher Aktivität auch im Rahmen von Alltagsbelastungen zu realisieren [34, 47].

\section{Krafttraining}

In der Vergangenheit wurde in der Prävention und Trainingstherapie internistischer Erkrankungen in erster Linie ein dynamisches aerobes Ausdauertraining empfohlen nun kommt bei Patienten mit Typ-2-Diabetes auch das Krafttraining zur Anwendung [47]. Es wird vorwiegend ein dynamisch-konzentrisches Krafttraining genutzt, da hierzu die meisten Studiendaten vorliegen. Bei der konzentrischen Kontraktionsform kommt es zu einer Verkürzung des arbeitenden Muskels mit einer Bewegung der beteiligten Gelenke. Empfohlen werden Übungen an 6-10 Muskelgruppen (Schultergürtel/Arme, Rumpf, Hüfte/Beine) pro Trainingseinheit. Bei der Wahl der Belastungsintensität wird derzeit davon ausgegangen, dass moderat-intensive Trainingsprotokolle die glykämische Stoffwechsellage am wirksamsten beeinflussen [47]. Empfohlen werden Belastungsintensitäten, die bei 75 $80 \%$ der individuellen Maximalkraft bzw. dem 1-RM (One Repetition Maximum) liegen. Allerdings ist es sinnvoll, gerade beim Einstieg ins Krafttraining zunächst niedrigere Intensitäten mit einer höheren Anzahl an Wiederholungen zu nutzen ( $\triangleright$ Tab. 2 ).

\section{Aerobes Ausdauertraining}

Beim aeroben Ausdauertraining wird neben der Dauermethode mittlerweile in wachsendem Maße auch die Methode des Intervalltrainings genutzt ( $\vee$ Tab. 3). Dabei gibt es Hinweise, dass Intervalltraining im Vergleich zu moderat-intensivem Ausdauertraining einerseits bei Pa- 
- Tab. 2 Empfehlungen zum konzentrischen Krafttraining bei Patienten mit Typ-2-Diabetes mit Angaben zu Belastungsintensität und -umfang. Einordnung der relativen Belastungsintensität in Bezug zur ermittelten individuellen Maximalkraft bei der jeweiligen Übung (1-RM, One Repetition Maximum). Die Ansteuerung der Belastungsintensität kann auch anhand der pro Übung noch ausführbaren Wiederholungszahl unter entsprechender Adjustierung des gewählten Gewichts erfolgen (modifiziert und ergänzt nach [12, 47, 48]).

\section{Krafttraining}

Durchführung in Form eines gerätegestützten Krafttrainings, unter Nutzung von freien Gewichten und/oder Widerstandsbändern. Wichtig ist die Einführung in die Übungstechnik und Überwachung der korrekten Durchführung, um eine optimale Wirksamkeit zu erreichen und Verletzungsrisiken zu minimieren.

Niedrig-intensives Krafttraining

- zum Trainingseinstieg

- als Kraftausdauertraining

- zum Übergang in ein moderat-intensives Krafttraining

Moderat-intensives Krafttraining

\section{Empfehlungen zu Intensität und Umfang}

\begin{tabular}{|l|l|l|}
$\begin{array}{l}\text { noch mögliche Wieder- } \\
\text { holungen pro Übung }\end{array}$ & $\%$ 1-RM & $\begin{array}{l}\text { Sets pro Muskel- } \\
\text { gruppe }\end{array}$ \\
\hline
\end{tabular}

tienten mit kardiometabolen Erkrankungen deutlicher die körperliche Fitness steigert [50]. Andererseits verringert Intervalltraining stärker das kardiometabole Risikoprofil [51].

Dies gilt auch für Patienten, die körperliche Aktivitätsprogramme als eher unangenehm empfinden. So berichten Adams et al. [52] für Patienten mit Claudicatio intermittens nach einem Aktivitätsprogramm mit kurzen hochintensiven Belastungsphasen nicht nur eine allgemeine Verbesserung des Rehabilitationsscores - darüber hinaus wurde das Programm sehr gut toleriert und es traten keine nachteiligen Effekte auf.

\section{Unterschiedliche Adaptationsprofile}

Kraft- und aerobes Ausdauertraining führen jeweils zu einem unterschiedlichen Muster der Reizeinwirkung. Dadurch induzieren sie auch ein differentes Adaptationsprofil in den sich anpassenden Gewebestrukturen, Körperfunktionen und im Risikoprofil ( Tab.4). So geht ein Ausdauertraining mit einem höheren Energieumsatz einher, steigert deutlicher die aerobe Kapazität und führt auch zu einer ausgeprägteren Anpassung der kardiovaskulären Funktion. Beim Krafttraining steht eine Zunahme des Muskelquerschnitts und der Kraftentwicklung im Vordergrund. Beide Trainingsformen führen aber zu einer weitgehend gleichen Verbesserung der glykolytischen Stoffwechsellage. Eine Kombination von Kraft- und Ausdauertraining bei Typ-2-Diabetes scheint sogar einen additiven Benefit zu erbringen [34].

\section{Merke}

Die Aktivitäts- und Trainingsempfehlungen der Leitlinien können durch Krafttraining und durch aerobes Ausdauertraining umgesetzt werden. Eine Kombination beider Trainingsformen scheint besonders günstig zu sein.

\section{Umsetzung des körperlichen Trainings}

Eine wirksame Trainingsdurchführung auch bei Patienten mit Typ-2-Diabetes leidet nicht selten unter einer verringerten Adhärenz [54]. Eine erfolgreiche, sichere und nachhaltige Trainingsdurchführung erfordert eine auf den Patienten abgestimmte personalisierte Trainingsplanung und -beratung mit einer klar formulierten Zielsetzung. Dabei geht es nicht nur um die Auswahl der Bewegungsform, sondern auch um die individuelle Beratung, mit welcher Belastungsintensität trainiert werden soll.

\section{Konzeptionierung des Trainingsprogramms}

Grundlage für eine personalisierte Trainingsplanung und -beratung sind die im Rahmen der Anamnese und Diagnostik erhobenen Informationen und Befunde. Die Konzeptionierung des Trainingsprogramms sollte sich dabei an den in $\mathbf{A b b} \mathbf{1}$ dargestellten Punkten orientieren.

\section{Begleit- und Folgeerkrankungen}

Es ist wichtig, neben dem Typ-2-Diabetes zusätzlich auch bekannte andere Erkrankungen oder Risikofaktoren zu beachten. Beispielsweise sollte bei begleitender Adipositas mit erforderlicher Gewichtsreduktion einem zusätzlichen Energieumsatz durch einen größeren Anteil an dynamischen Ausdauerbelastungen Rechnung getragen werden.

Von grundlegender Bedeutung ist es, dass die körperliche Belastbarkeit des Patienten bekannt ist und mögliche Risiken durch Begleit- oder Folgeerkrankungen beachtet werden. Insbesondere Befunde im Bereich des kardiovaskulären Systems, des Haltungs- und Bewegungsapparates und diabetesspezifische Komplikationen sollten vor Beginn des Trainings erfasst werden. Wenn erforderlich, ist das Training dann darauf abzustimmen. 
- Tab. 3 Formen des dynamischen Ausdauertrainings mit Angaben der korrespondierenden Belastungsintensität. Einordnung der relativen Belastungsintensität anhand des subjektiven Belastungsempfindens nach BORG-Skala, in Bezug zur individuellen maximalen Herzfrequenz (\% $\mathrm{HF}_{\max }$ ) und zur maximalen Sauerstoffaufnahme $\left(\% \mathrm{VO}_{2 \max }\right)$ sowie in Relation zu den leistungsdiagnostischen Eckpunkten Lactate Threshold (LT, entspricht dem Beginn des Laktatanstiegs im ergometrischen Stufentest), individuelle anaerobe Schwelle (IAS) und maximale Sauerstoffaufnahme $\left.\mathrm{VO}_{2 \max }\right)($ modifiziert und ergänzt nach $[24,47,49])$.

\begin{tabular}{|c|c|c|c|c|c|}
\hline \multicolumn{2}{|l|}{ Dynamisches Ausdauertraining } & \multicolumn{4}{|c|}{ Relative Belastungsintensität } \\
\hline $\begin{array}{l}\text { Typische Formen des dynamischen } \\
\text { Ausdauertrainings sind Gehen } \\
\text { (Walking), Laufen, Radfahren, } \\
\text { Schwimmen, Rudern, Skilanglauf } \\
\text { und bei Intervalltraining zusätzlich } \\
\text { auch Spielsportarten wie z. B. } \\
\text { Fußball, Basketball oder Tennis }\end{array}$ & Intensitätsstufe & $\begin{array}{l}\text { subjektives } \\
\text { Belastungsemp- } \\
\text { finden (nach } \\
\text { BORG-Skala, } \\
6-20 \text { ) }\end{array}$ & $\% \mathrm{HF}_{\max }$ & $\% \mathrm{VO}_{\max }{ }^{1}$ & $\begin{array}{l}\text { Bezug zu leistungs- } \\
\text { diagnostischen } \\
\text { Eckpunkten }\end{array}$ \\
\hline \multirow{2}{*}{$\begin{array}{l}\text { Aerobes Ausdauertraining: Durch- } \\
\text { führung nach der Dauermethode mit } \\
\text { dynamischen Belastungen mehrerer } \\
\text { größerer Muskelgruppen von min- } \\
\text { destens } 10 \text { Minuten Dauer ohne Un- } \\
\text { terbrechung. Dabei kommt es zu kei- } \\
\text { ner verstärkten Laktatakkumulation. }\end{array}$} & moderat & $12-13$ & $55-69$ & $40-59$ & $\leq \mathrm{LT}$ \\
\hline & anstrengend & $14-16$ & $70-89$ & $60-84$ & zwischen LT und IAS \\
\hline \multirow[b]{2}{*}{$\begin{array}{l}\text { Intervalltraining: Durchführung in } \\
\text { Form mehrerer Belastungsabschnitte } \\
\text { (Intervalle), die von Pausen mit nied- } \\
\text { riger Belastungsintensität unterbro- } \\
\text { chen werden. Beispiele: } 5 \times 4 \text { min } \\
\text { Belastung mit } 3 \text { min Pause oder } \\
8 \times 2 \text { min mit } 2 \text { min Pause mit } \\
\text { zuvor erfolgter Aufwärmphase } \\
(10-15 \text { min) mit niedriger Intensität. }\end{array}$} & anstrengend & $14-16$ & $70-89$ & $60-84$ & zwischen LT und IAS \\
\hline & sehr anstrengend & $17-19$ & $\geq 90$ & $85-99$ & $\geq$ IAS \\
\hline
\end{tabular}

\section{Individuelle Leistungsfähigkeit}

Auch die körperliche Leistungsfähigkeit zeigt eine ausgesprochen große individuelle Variabilität - dies gilt es bei der Trainingsgestaltung, insbesondere bei Neueinsteigern in ein Bewegungs- und Trainingsprogramm, zu berücksichtigen. Objektiviert werden kann die körperliche Leistungsfähigkeit mittels ergometrischer Verfahren.

\section{Merke}

Damit die Patienten die definierten Trainingsziele erreichen können, sollte das Training eine ausreichende Wirksamkeit aufweisen.

Die Wahl einer niedrigeren Belastungsintensität hat den Vorteil, dass die Belastungsdauer länger aufrechterhalten werden kann. Die Belastung des Bewegungsapparats ist dabei geringer und dem Patienten fällt der Trainingseinstieg somit häufig leichter. Andererseits hat ein intensiveres, ggf. auch intervallartig betriebenes Training oder aktiv betriebener Spielsport den Vorteil, dass die körperliche Fitness rascher gesteigert werden kann. Auch weisen diese Belastungsformen in der Regel eine höhere Zeiteffizienz auf.
Bei wenig belastbaren Patienten und/oder gering leistungsfähigen Neueinsteigern muss die Belastungsgestaltung zu Beginn behutsam gesteigert werden. Dabei können zunächst kurze Trainingszeiten von 5-10 min gewählt werden. Diese können dann im Verlauf auch mehrfach über den Tag verteilt durchgeführt werden, um einen ausreichenden Umfang zu erreichen [47].

Vorerfahrungen in bestimmten Sportarten oder Bewegungsformen sind hilfreich, um in ein Trainingsprogramm einzusteigen bzw. dieses zu optimieren. Dies gilt insbesondere auch für Sportarten, die eine erlernte Technik erfordern, wie beispielsweise das Schwimmen.

\section{FALLBEISPIEL}

Eine 69-jährige Patientin mit einem vor 9 Jahren diagnostizierten Typ-2-Diabetes und einem BMI von $28,7 \mathrm{~kg} / \mathrm{m}^{2}$ stellt sich zur Einleitung einer Trainingstherapie vor. An diabetesbedingten Folgeschäden liegt eine milde nicht proliferative Retinopathie vor. Begleitdiagnosen sind arterielle Hypertonie, Hypercholesterinämie sowie Gonarthrose Grad I bds. Die Medikation umfasst Metformin, einen ACE-Hemmer und ein Statinpräparat. Um die Belastbarkeit und 
- Tab. 4 Vergleich der Effekte von Ausdauer- und Krafttraining auf ausgewählte muskuläre, metabolische und kardiozirkulatorische Zielvariablen (modifiziert und ergänzt nach [48, 53]).

\begin{tabular}{|l|l|l|}
\hline Variable & $\begin{array}{l}\text { Aerobes } \\
\text { Ausdauertraining }\end{array}$ & $\begin{array}{l}\text { Kraft- } \\
\text { training }\end{array}$ \\
\hline Maximale Sauerstoffaufnahme & $\uparrow \uparrow$ & $\leftrightarrow / \uparrow$ \\
\hline $\begin{array}{l}\text { Leistung an der individuellen } \\
\text { anaeroben (Laktat-)Schwelle }\end{array}$ & $\uparrow \uparrow \uparrow$ & $\leftrightarrow / \uparrow$ \\
\hline Körperfettanteil (\%) & $\downarrow \downarrow$ & $\downarrow$ \\
\hline Fettfreie Körpermasse & $\leftrightarrow / \uparrow$ & $\uparrow \uparrow$ \\
\hline Muskelkraft & $\leftrightarrow / \uparrow$ & $\uparrow \uparrow \uparrow$ \\
\hline $\begin{array}{l}\text { Ektope Fettspeicher (Leber, } \\
\text { viszeral) }\end{array}$ & $\downarrow \downarrow \downarrow$ & $\leftrightarrow / \downarrow$ \\
\hline Insulinsensitivität & $\uparrow \uparrow$ & $\uparrow \uparrow$ \\
\hline HDL-Cholesterin & $\uparrow$ & $\leftrightarrow / \uparrow$ \\
\hline LDL-Cholesterin & $\leftrightarrow / \downarrow$ & $\leftrightarrow / \downarrow$ \\
\hline Ruheumsatz & $\uparrow$ & $\uparrow$ \\
\hline Herz-Kreislauf-System: & & $\leftrightarrow / \downarrow$ \\
\hline - Ruheherzfrequenz & $\downarrow \downarrow$ & $\leftrightarrow$ \\
\hline - Schlagvolumen & $\uparrow \uparrow$ & $\downarrow$ \\
\hline - Ruheblutdruck (systolisch) & $\downarrow$ & $\downarrow$ \\
\hline - Ruheblutdruck (diastolisch) & $\downarrow$ & \\
\hline
\end{tabular}

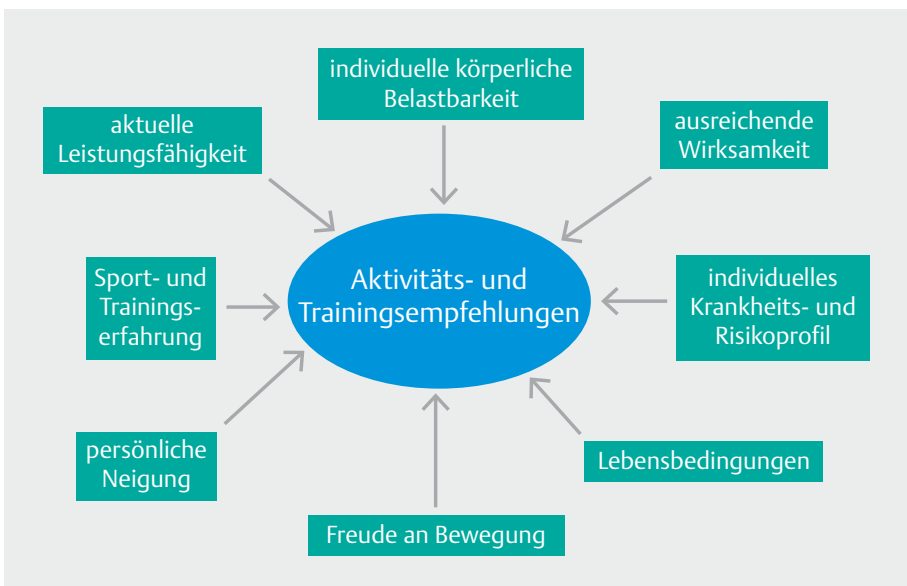

- Abb. 1 Bei der personalisierten Trainingsberatung zu beachtende Kriterien.

Leistungsfähigkeit zu klären, erfolgt eine stufenförmige Fahrradergometrie mit Belastungs-EKG und Blutdruckmonitoring. Bei einer maximalen, unter subjektiver Ausbelastung erreichten Leistung von 100 Watt finden sich ein unter Medikation regelrechtes Blutdruckverhalten und EKG.

Der Patientin wird ein fahrradergometrisches Training von zunächst 2 Einheiten/Woche über 30 40 min empfohlen (bei 60 - $70 \%$ der im Ergometer- test ermittelten max. Herzfrequenz); bei im Verlauf guter Tolerierung ergänzend/alternativ Radfahren oder Walking im Freien. Das Ausdauertraining soll dann schrittweise auf 3 Einheiten/Woche à 45 60 min erhöht werden. Auch die Belastungsintensität kann bis auf $80 \%$ der max. Herzfrequenz gesteigert werden, wenn sich die Patientin dabei gut fühlt.

Zusätzlich wird der Beginn eines gerätegestützten Kraftausdauertrainings unter Anleitung empfohlen, bei dem jeweils $5-6$ große Muskelgruppen in 2 Durchgängen beübt werden. Das Trainingsprogramm soll ergänzt werden durch Alltagsaktivitäten (Wegstrecken zu Fuß zurücklegen, Arbeiten im Haushalt) sowie das Meiden längerer Sitzphasen.

\section{Motivation und Beratung}

Lebensbedingungen

Wichtig ist, das körperliche Training in den Alltag zu implementieren. Dabei müssen insbesondere die Lebensbedingungen des Patienten erfragt werden. So ist es nicht jedem Patienten möglich, regelmäßig zu bestimmten Zeitpunkten ein Training durchzuführen. Auch leben manche Patienten in Umgebungen, die infrastrukturell nicht besonders bewegungsförderlich sind. Zudem verfügen die Patienten häufig nicht über soziale Netzwerke, die zur regelmäßigen Bewegung animieren. Gerade den individuellen sozialen Netzwerken sollte mit Blick auf eine nachhaltige Implementierung von Bewegung in den Alltag in der Trainingstherapie noch mehr Aufmerksamkeit gewidmet werden. So haben Carrell et al. [55] nachgewiesen, dass Inaktivität und schlechte Fitness „sozial ansteckend“ sind. Die Ausbreitung von schlechter körperlicher Fitness in sozialen Netzwerken führen sie darauf zurück, dass Menschen die Ernährungs- und Bewegungsgewohnheiten ihrer am wenigsten fitten Freunde imitieren. Die Fitness ihres Umfeldes nutzen sie dabei als eine Art „Benchmark“ für sich selbst. Diese Beobachtung verweist auf ein „Unterstützungs-Dilemma“. Denn einerseits können soziale Netzwerke zwar das Inaktivitätsrisiko steigern. Andererseits ist die aktive soziale Unterstützung insbesondere die Einbeziehung von Familienangehörige in das Programm [56] - aber besonders förderlich für die Erhöhung des Aktivitätsumfangs.

\section{Persönliche Neigungen und Barrieren}

Risikogruppen sind Menschen mit einer geringen Selbstwirksamkeit und einer geringen Bereitschaft, ihren Lebensstil zugunsten von mehr körperlicher Aktivität zu ändern. Bei ihnen ist individuelle Beratung besonders wichtig. So zeigt eine ganze Reihe an gesundheitspsychologischen Analysen bei Diabetes-Patienten: Eine hohe Selbstwirksamkeit und eine motivationale Bereitschaft zur körperlichen Aktivität stellen gute Prädiktoren für die Adhärenz bei Aktivitätsprogrammen dar [57, 58]. 
Beim Anpassen von Programmen an persönliche Neigungen ist zu beachten, dass Männer einer medizinisch motivierten Änderung des Lebensstils generell weniger zugeneigt sind als Frauen [59]. Empfohlen wird daher, die Ziele eines Trainingsprogramms geschlechtsspezifisch zu definieren. Bei Männern sollte man beispielsweise das Erreichen eines gewissen Fitnesslevels sowie einer maskulinen Körperform in den Mittelpunkt stellen. Frauen können dagegen durch gesundheitsbezogene Zielsetzungen recht gut erreicht werden.

\section{Merke}

Hinsichtlich der Motivation der Patienten für Trainingsprogramme empfiehlt es sich, auch geschlechtsspezifische Neigungen bei der Definition der Trainingsziele zu berücksichtigen.

In einem systematischen Review [60] wurde zwischen externalen und internalen Aktivitätsbarrieren bei Patienten mit Typ-2-Diabetes unterschieden. Als externale Barrieren wurden schwierige Lebensverhältnisse, Wetter, ein Mangel an erreichbaren Sportstätten sowie fehlende soziale Unterstützung genannt. Internale Barrieren umfassten Zeitmanagement, ein schlechtes subjektives Kosten-Nutzen-Verhältnis von Aufwand und Ertrag körperlicher Aktivität, aber auch Emotionen wie Scham, Faulheit und Mangel an Spaß am körperlichen Training [60]. Internale und externale Faktoren wirken dabei teilweise zusammen, beispielsweise in klimatisch ungünstigen und wenig besiedelten Regionen [61].

\section{Freude an der Bewegung}

Freude an der körperlichen Aktivität wird in verschiedenen Studien als relevanter Faktor der Adhärenz bei Aktivitätsprogrammen für Patienten mit Typ-2-Diabetes genannt [62]. Diesem Aspekt wird bei der Konzeption von Interventionsprogrammen häufig zu wenig Bedeutung zugemessen. In der Praxis stellt gesundheitsförderliches körperliches Training im Erwachsenenalter tendenziell eine eher funktionale und isolierte Maßnahme dar. Deshalb ist es für bestimmte Risikogruppen, wie z. B. Adipöse, vermutlich eher unattraktiv [63].

Etwas mehr Aufmerksamkeit wurde in vorliegenden Studien der Frage gewidmet, ob Patienten kurze intensive oder länger andauernde moderate Belastungseinheiten als angenehmer empfinden. Die Studien zeichnen hier allerdings ein uneinheitliches Bild. Bartlett et al. [64] kommen zu dem Schluss, dass ein hochintensives IntervallLauftraining angenehmer ist als ein länger andauerndes moderates Training. Dagegen berichten Foster et al. [65], dass ein hochintensives Intervallprogramm im Vergleich zu einem moderaten Steady-State-Ausdauertraining von den Teilnehmern als unangenehmer wahrgenommen wurde. Einschränkend ist allerdings anzumerken, dass sich in der letztgenannten Studie bei allen Trainingsfor- men nach einer Weile eine Abnahme der Freude an der Aktivität zeigte.

\section{PRAXISTIPP}

Qualifizierte Betreuer

Insgesamt ist festzuhalten, dass bei der Konzipierung und Umsetzung körperlichen Trainings für DiabetesPatienten der motivationalen Aktivitätsbereitschaft und Selbstwirksamkeit der Patienten besondere Aufmerksamkeit zu widmen ist. Dem betreuenden Personal kommt deshalb sowohl für die Konzipierung, Umsetzung und Evaluation des Trainingsprogramms als auch für die Beratung der Patienten zentrale Bedeutung zu. Als sinnvoll haben sich in gesundheitswissenschaftlichen Evaluationsstudien für die Beratung Einzel- und Gruppengespräche erwiesen zur Förderung von Ziel- und Implementierungsintentionen sowie volitionaler Selbstregulationsfertigkeiten. Wie wichtig die personalisierte Trainingsplanung und -beratung durch einen qualifizierten Betreuer ist, zeigten Kirk et al. [66]: Trainingsberatung bei Patienten mit Typ-2-Diabetes war mit erhöhter körperlicher Aktivität, verbesserter glykämischer Kontrolle und einem günstigeren kardiovaskulären Risikoprofil assoziiert.

\section{Risiken und körperliche Belastbarkeit}

Sowohl eine unzureichende glykämische Kontrolle als auch Folgeschäden der Diabeteserkrankung können ein Risiko für den Patienten darstellen und dessen körperliche Belastbarkeit einschränken. Aus diesem Grund wird vor Beginn eines körperlichen Trainings eine sorgfältige Eingangsdiagnostik empfohlen.

\section{Retinopathie}

Bei einer proliferativen Retinopathie oder einer schweren nicht proliferativen Retinopathie wird empfohlen, auf Belastungen zu verzichten, die zu einem stärkeren Blutdruckanstieg führen. Um das damit verbundene Risiko einer Netzhautablösung oder einer Glaskörperblutung zu verringern, sollten höher-intensive aerobe Ausdauerbelastungen und Krafttraining vermieden werden. Dies gilt insbesondere für Belastungen, bei denen es zu Valsalva-Manövern kommt [34, 47]. Je nach Ausprägung sollten regelmäßige Verlaufsuntersuchungen der Retinopathie vorgenommen werden und auf eine gute Blutdruckeinstellung geachtet werden.

Vorsicht

Bei Retinopathie müssen stärkere Blutdruckanstiege und Valsalva-Manöver im Rahmen des Trainings vermieden werden. 


\section{Periphere Neuropathie}

Infolge einer verringerten Schmerzwahrnehmung kann es bei einer peripheren Neuropathie zu Verletzungen und Infektionen an der Haut des Fußes sowie zur Entwicklung eines Charcot-Gelenkschadens kommen. Ein moderat-intensives Ausdauertraining in Form von Walking erhöht bei Patienten mit Typ-2-Diabetes und nachgewiesener peripherer Neuropathie aber wohl nicht das Risiko für diese Folgeschäden - es scheint sogar die neuropathischen Beschwerden abzumildern [64]. In jedem Falle ist bei Vorliegen einer peripheren Neuropathie auf eine optimale Schuhversorgung zu achten. Tägliche Fußkontrollen zum frühen Erkennen von Hautläsionen sind durchzuführen. Bei offenen Hautverletzungen muss auf fußbelastende Aktivitäten verzichtet werden [34, 47].

\section{Autonome Neuropathie}

Im Falle einer autonomen Neuropathie steigt nicht nur das Risiko für Hypoglykämien. Darüber hinaus besteht auch ein erhöhtes Risiko für einen inadäquaten Herzfrequenz- und Blutdruckanstieg bei körperlicher Belastung, was zu einer Belastungsintoleranz beitragen kann [34]. Ebenso kann eine kardiale autonome Neuropathie das Auftreten stummer Myokardischämien und kardialer Zwischenfälle begünstigen [68]. Eine weitere Folge der autonomen Neuropathie ist eine gestörte Thermoregulation sowohl bei Hitze als auch in der Kälte [69]. Bei Patienten mit Verdacht auf eine autonome Neuropathie sollte eine engere kardiologische Diagnostik erfolgen, auf deren Basis die Aktivitäts- und Trainingsempfehlungen anzupassen sind [34, 47]. Gegebenenfalls müssen die Belastungsintensitäten im Training reduziert und Aktivitäten bei hohen Umgebungstemperaturen gemieden werden [47].

\section{Albuminurie und Nephropathie}

Es ist bekannt, dass eine akute körperliche Belastung zu einer Zunahme der Proteinausscheidung führen kann. Allerdings gibt es keine Hinweise dafür, dass selbst intensivere körperliche Belastung zu einer Progression der diabetischen Nephropathie beitragen kann. Eine Einschränkung der körperlichen Aktivität wird in diesen Fällen nicht gesehen $[34,47]$.

\section{Koronare Herzerkrankung}

Aufgrund des erhöhten Risikos für eine koronare Herzerkrankung sollte im Rahmen der Vordiagnostik die Indikation für ein Belastungs-EKG großzügig gestellt werden. Im Consensus Report der ADA wird zwar kein RoutineScreening mittels Belastungs-EKG bei Patienten mit Typ2-Diabetes empfohlen [70]. Jedoch wird ein BelastungsEKG dann als sinnvoll angesehen, wenn die körperliche Belastung den moderaten Intensitätsbereich überschreitet und/oder weitere Risikofaktoren vorliegen [71]. Die S1-Leitlinie der Deutschen Gesellschaft für Sportmedizin und Prävention (DGSP) empfiehlt ein Belastungs-EKG bei
Männern über 40 und Frauen über 50 Jahren vor Beginn intensiver körperlicher Belastung, wenn einer oder mehr kardiovaskuläre Risikofaktoren vorliegen [71]. Damit erfüllt ein größerer Teil der Zielgruppe von Patienten mit Typ-2-Diabetes die Indikationskriterien. Eine Ergometrie mit zusätzlicher Laktatdiagnostik und/oder Spiroergometrie ermöglicht es,

- den Belastungsblutdruck und Ischämiezeichen im Belastungs-EKG zu erfassen,

- die körperliche Fitness präziser zu objektivieren und

- darüber hinaus auch individuelle Intensitätsempfehlungen für das dynamische Ausdauertraining zu ermitteln [72].

\begin{abstract}
Merke
Die Indikation für ein Belastungs-EKG sollte bei Patienten mit Typ-2-Diabetes vor dem Beginn eines körperlichen Trainings großzügig gestellt werden.
\end{abstract}

\section{Hyperglykämie und Ketose}

Besteht ein Insulinmangel, kann körperliche Belastung zu einer Verschärfung der Stoffwechsellage führen. Dabei tragen die erhöhte hepatische Glukoseproduktion und die belastungsinduzierte Ausschüttung kontrainsulinärer Hormone zu einem Anstieg der Plasmaglukose bei - diese gelangt infolge des Insulinmangels jedoch nicht in die arbeitende Muskelzelle. Gleichzeitig verursacht das daraus resultierende Energiedefizit im arbeitenden Skelettmuskel eine weitere Steigerung der Lipolyse mit dem Risiko der Entstehung einer Ketose [53]. Bei Blutzuckerwerten über $300 \mathrm{mg} / \mathrm{dl}$ sollten Ketone im Urin oder Blut bestimmt werden. Bei positivem Nachweis ist zunächst auf intensivere Belastungen zu verzichten [47]. Eine alleinige Hyperglykämie ohne Nachweis einer Ketose und ohne klinische Symptomatik wird bei Patienten mit Typ2-Diabetes demgegenüber nicht als Kontraindikation für körperliche Belastungen angesehen [34, 47]. Allerdings sollte in dieser Situation auf eine ausreichende Flüssigkeitszufuhr und auf das mögliche Auftreten klinischer Symptome geachtet werden.

\section{Hypoglykämie}

Körperliche Belastung steigert die muskuläre Glukoseaufnahme auch durch insulinunabhängige Mechanismen, was zu einem Abfall der Plasmaglukose führen kann, wenn gleichzeitig noch eine Insulinwirkung besteht. Im Gegensatz zu Patienten mit Typ-1-Diabetes ist das Risiko für eine belastungsinduzierte Hypoglykämie bei Patienten mit Typ-2-Diabetes geringer ausgeprägt. Allerdings kann auch bei diesen unter Medikation mit Insulin oder Sekretagoga - z. B. Glinide und Sulfonylharnstoffe - ein Blutzuckerabfall auftreten, wenn die Medikation und/ oder die Kohlenhydratzufuhr nicht angepasst werden. Unter einer entsprechenden Medikation sollten zusätzliche Blutzuckerkontrollen durchgeführt werden, um das individuelle Vorgehen bei der Vermeidung von Hypogly- 
kämien steuern zu können. Beachtet werden muss: Es kann auch erst mehrere Stunden nach Belastung und nach einer abendlichen Trainingseinheit auch nachts zu einem Abfall des Blutzuckers kommen. Wird der Blutzucker mit Lebensstilmaßnahmen und/oder Antidiabetika, die nicht zu einer Insulinerhöhung führen, eingestellt, ist das Auftreten einer belastungsinduzierten Hypoglykämie demgegenüber unwahrscheinlich [12].

\section{Anpassung der Insulindosierung und Kohlenhydratgabe}

Patienten, die mit Insulin oder Sekretagoga behandelt werden, sollten im Falle von Blutzuckerwerten unter $100 \mathrm{mg} / \mathrm{dl}$ vor Belastungsbeginn 15 - $30 \mathrm{~g}$ Kohlenhydrate zuführen. Hinsichtlich der Insulindosierung wird vor moderat-intensiven Trainingsbelastungen von 30-60 min Dauer eine Reduktion der Insulindosis um 25 - 75 \% empfohlen. Wird am Morgen trainiert, sollte bereits die vorabendliche Gabe das Basalinsulins um 20 - $50 \%$ verringert werden. Beginnt die Trainingsbelastung mehr als 3 Stunden nach der letzten Insulininjektion und Mahlzeit, sollte die Dosis des Insulins nicht verringert werden. Stattdessen sollte ggf. mit der Gabe von Kohlenhydraten gearbeitet werden. Sind intensivere und/oder längere Trainingsbelastungen geplant, muss die Insulindosis ggf. noch weiter reduziert werden $[47,53]$. In einer Untersuchung an insulinbehandelten Patienten mit Typ-2-Diabetes wurde die Insulindosis vor einer Trainingsbelastung um $50 \%$ reduziert. Sowohl nach einem Kraft- als auch einem moderat-intensiven Ausdauertraining über 30 min war keine anschließende hyper- oder hypoglykämische Reaktion zu beobachten [73]. Auch bei Behandlung mit Sekretagoga kann im Rahmen eines körperlichen Trainings eine Dosisreduktion notwendig werden.

\section{Merke}

Unter der Medikation mit Insulin oder Sekretagoga müssen bei körperlichem Training häufigere Blutzuckerkontrollen und ggf. Dosisanpassungen der Medikation erfolgen und/oder Kohlenhydrate zugeführt werden.

\section{KERNAUSSAGEN}

- Körperliche Aktivität zählt zu den wichtigsten nicht medikamentösen Maßnahmen zur Prävention und Therapie des Typ-2-Diabetes.

- Patienten mit Typ-2-Diabetes profitieren von körperlicher Aktivität nicht nur in Hinblick auf eine verbesserte glykämische Kontrolle. Weitere positive Effekte sind die Absenkung kardiovaskulärer Risikofaktoren, die Verringerung ektoper Fettdepots, eine Gewichtsreduktion, eine antiinflammatorische Wirkung und eine Verbesserung und Stabilisierung der körperlichen Fitness.

- Ein ausreichender Umfang an körperlicher Aktivität reduziert bei Patienten mit Typ-2-Diabetes sowohl die Gesamtmortalität als auch die kardiovaskuläre Sterblichkeit.

- Patienten mit Typ-2-Diabetes wird empfohlen, mindestens 150 min pro Woche an moderat-intensiven aeroben Ausdauerbelastungen zu absolvieren und das Training auf mindestens 3 Tage pro Woche zu verteilen.

- Sofern keine Kontraindikationen bestehen, wird Erwachsenen zusätzlich ein Krafttraining mit zumindest 2 Trainingseinheiten pro Woche empfohlen.

- Um die individuelle Belastbarkeit zu klären, wird bei Patienten mit Typ-2-Diabetes vor Beginn eines körperlichen Trainings eine sorgfältige Eingangsdiagnostik empfohlen.

- Bei trainierenden Patienten mit Typ-2-Diabetes, die mit Insulin oder Sekretagoga behandelt werden, sollte je nach individueller Konstellation die Medikation angepasst werden.

- Im Falle von Blutzuckerwerten unter 100 mg/dl vor Belastungsbeginn sollten zusätzlich Kohlenhydrate zugeführt werden.

\section{Schlüsselwörter}

Typ-2-Diabetes, aerobes Ausdauertraining, Krafttraining, glykämische Kontrolle, sedenter Lebensstil

Key words

type 2 diabetes, aerobic exercise training, resistance training, glycemic control, sedentariness

\section{Zusammenfassung}

Ein ausreichender Umfang an körperlicher Aktivität zählt zu den wichtigsten nicht medikamentösen Maßnahmen zur Prävention und Therapie des Typ-2-Diabetes. Von einem regelmäßigen körperlichen Training profitieren Patienten mit Typ2-Diabetes nicht nur in Hinblick auf eine verbesserte glykämische Kontrolle. Weitere positive Wirkungen sind eine günstige Beeinflussung kardiovaskulärer Risikofaktoren, eine Verringerung ektoper Fettdepots, eine Unterstützung bei der Reduktion von Übergewicht, antiinflammatorische Effekte und eine Verbesserung und Stabilisierung der körperlichen Fitness. Somit senkt ein ausreichender Umfang an körperlichem Training sowohl die Gesamtmortalität als auch die kardiovaskuläre Sterblichkeit bei Typ-2-Diabetes.

Nach den Leitlinien zur Therapie des Typ-2-Diabetes sollen betroffene Erwachsene angehalten werden, mindestens 150 min pro Woche an moderat-intensiven aeroben Ausdauerbelastungen ( 50 - $70 \%$ der maximalen Herzfrequenz) zu absolvieren. Dieses Training sollte auf mindestens 3 Tage pro Woche verteilt sein und es sollten nicht mehr als 2 trainingsfreie Tage dazwischen liegen. Sofern keine Kontraindikationen bestehen, wird Erwachsenen mit Typ-2-Diabetes zusätzlich ein Krafttraining mit zumindest zweimaliger Durchführung pro Woche empfohlen. Des Weiteren sollten Phasen längeren Sit- 
zens vermieden werden und Sitzzeiten alle 90 min unterbrochen werden.

Sowohl eine unzureichende glykämische Kontrolle als auch Folgeschäden der Diabeteserkrankung wie eine Retinopthie, eine autonome Neuropathie oder eine koronare Herzerkrankung können im Rahmen des körperlichen Trainings für den Patienten ein Risiko darstellen und dessen körperliche Belastbarkeit einschränken. Aus diesem Grund wird vor Beginn eines körperlichen Trainings eine sorgfältige Eingangsdiagnostik und wenn notwendig eine Anpassung des Trainingsprogramms empfohlen. Weiterhin sollte bei trainierenden Patienten mit Typ-2-Diabetes unter Medikation mit Insulin oder Sekretagoga je nach individueller Konstellation die Medikation angepasst werden. Im Falle von Blutzuckerwerten unter $100 \mathrm{mg} / \mathrm{dl}$ vor Belastungsbeginn sollten zusätzlich Kohlenhydrate zugeführt werden.

Abstract

A sufficient amount of physical activity is an important tool to prevent and treat type 2 diabetes. Beside an improvement in glycemic control, beneficial effects of physical activity and exercise training include a reduction of the cardiovascular risk profile, body weight and ectopic fat stores, anti-inflammatory properties and also an increase of physical fitness. In this context, exercise training lowers all-cause and cardiac mortality in type 2 diabetes.

Actual recommendations reflect that adults with type $2 \mathrm{di}$ abetes should be advised to perform at least 150 min per week of moderate intensive aerobic exercise training (50-70\% maximum heart rate). The training activity should be spread over at least three sessions during a week, and resting periods between the sessions should not exceed two days. In addition, in the absence of contraindications, adults with type 2 diabetes should be encouraged to perform resistance training at a minimum of twice a week. Moreover, a reduction of sedentary time by breaking up periods of sitting longer than 90 minutes should be realized.

Risks during exercise training can arise from a non-optimal glycemic control. Moreover, long-term complications of diabetes such a retinopathy, autonomic neuropathy and coronary heart disease may lower tolerance to exercise. Thus, a pretraining evaluation and clinical judgement should be performed. If necessary, the training regimen has to be adapted to the individual case. To avoid hypoglycemia in patients treated with insulin or secretagogues, the medication dose has to be adapted individually and/or additional carbohydrates supplemented, if pre-exercise plasma glucose is measured below $100 \mathrm{mg} / \mathrm{dl}$.

\section{Interessenkonflikt}

Die Autoren geben an, dass kein Interessenkonflikt besteht.

\section{Über die Autoren}

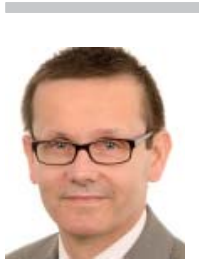

\section{Prof. Dr. med. Andreas M. Nieß}

Medizinstudium in Ulm, 1989 Approbation. 1990-2002 Facharztausbildung, 2002 Facharzt Innere Medizin, Sportmedizin, 2002 Habilitation an der Medizinischen Fakultät, Universität Tübingen. 2002-2004 Oberarzt Abteilung Rehabilitative \& Präventive Sportmedizin, Uniklinikum Freiburg. Seit 2004 Ärztlicher Direktor Abteilung Sportmedizin, Uniklinikum Tübingen. Schwerpunkte: Internistische Sportmedizin, Prävention, Trainingstherapie.

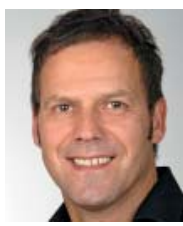

\section{Prof. Dr. phil. Ansgar Thiel}

Studium der Sportwissenschaft (Diplom) Psychologie und Psychogerontologie (Diplom) an den Universitäten Tübingen und Erlangen Nürnberg. 1996 Promotion, 2001 Habilitation an der Universität Bielefeld. Professor für Sportwissenschaft an der Universität Tübingen. Seit 2010 Direktor des Instituts für Sportwissenschaft. Schwerpunkte: Soziale Bedingungen körperlicher Aktivität, Gesundheit im Spitzensport.

\section{Korrespondenzadresse}

Prof. Dr. med. Andreas M. Nieß

Abteilung Sportmedizin

Medizinische Klinik

Universitätsklinikum Tübingen

Hoppe-Seyler-Straße 6

72076 Tübingen

andreas.niess@med.uni-tuebingen.de

\section{Literatur}

[1] Lindström J, Peltonen M, Eriksson JG et al. Finnish Diabetes Prevention Study (DPS). Improved lifestyle and decreased diabetes risk over 13 years: long-term follow-up of the randomised Finnish Diabetes Prevention Study (DPS). Diabetologia 2013; 56: 284-293

[2] Umpierre D, Ribeiro PA, Schaan BD et al. Volume of supervised exercise training impacts glycaemic control in patients with type 2 diabetes: a systematic review with meta-regression analysis. Diabetologia 2013; 56: 242-251

[3] Yang Z, Scott CA, Mao C et al. Resistance exercise versus aerobic exercise for type 2 diabetes: a systematic review and meta-analysis. Sports Med 2014; 44: 487-499

[4] Hu G, Jousilahti P, Barengo NC et al. Physical activity, cardiovascular risk factors, and mortality among Finnish adults with diabetes. Diabetes Care 2005; 28: 799-805

[5] Ratner R, Goldberg R, Haffner S, Diabetes Prevention Program Research Group et al. Impact of intensive lifestyle and metformin therapy on cardiovascular disease risk factors in the diabetes prevention program. Diabetes Care 2005; 28: 888 894

[6] Look AHEAD Research Group. Cardiovascular effects of intensive lifestyle intervention in type 2 diabetes. $N$ Engl J Med 2013; 369: 145-154

[7] Rejeski W], Ip EH, Bertoni AG, Look AHEAD Research Group et al. Lifestyle change and mobility in obese adults with type 2 diabetes. N Engl ] Med 2012; 366: 1209-1217 
[8] Hallal PC, Andersen LB, Bull FC et al. Global physical activity levels: surveillance progress, pitfalls, and prospects. Lancet 2012; 380: $247-257$

[9] Nelson KM, Reiber G, Boyko EJ, NHANES III. Diet and exercise among adults with type 2 diabetes: findings from the third national health and nutrition examination survey (NHANES III). Diabetes Care 2002; 25: 1722 - 1728

[10] Ekelund U, Steene-Johannessen J, Brown W, Lancet Physical Activity Series 2 Executive Committe; Lancet Sedentary Behaviour Working Group et al. Does physical activity attenuate, or even eliminate, the detrimental association of sitting time with mortality? A harmonised meta-analysis of data from more than 1 million men and women. Lancet 2016; 388: $1302-1310$

[11] Biswas A, Oh PI, Faulkner GE et al. Sedentary time and its association with risk for disease incidence, mortality, and hospitalization in adults: a systematic review and meta-analysis. Ann Intern Med 2015; 162: 123-132

[12] Colberg SR, Sigal RJ, Fernhall B, American College of Sports Medicine; American Diabetes Association et al. Exercise and type 2 diabetes: the American College of Sports Medicine and the American Diabetes Association: joint position statement executive summary. Diabetes Care 2010; 33: 2692 - 2696

[13] Kessler HS, Sisson SB, Short KR. The potential for high-intensity interval training to reduce cardiometabolic disease risk. Sports Med 2012; 42: 489 - 509

[14] Henriksen EJ. Invited Review: Effects of acute exercise and exercise training on insulin resistance. J Appl Physiol 2002; 93: $788-796$

[15] Jørgensen SB, Richter EA, Wojtaszewski JF. Role of AMPK in skeletal muscle metabolic regulation and adaptation in relation to exercise. J Physiol 2006; 574: 17-31

[16] Kramer HF, Goodyear LJ. Exercise, MAPK, and NF-kappaB signaling in skeletal muscle. J Appl Physiol 2007; 103: 388 - 395

[17] Bruce CR, Hawley JA. Improvements in insulin resistance with aerobic exercise training: a lipocentric approach. Med Sci Sports Exerc 2004; 36: 1196 - 1201

[18] Kantartzis K, Thamer C, Peter A et al. High cardiorespiratory fitness is an independent predictor of the reduction in liver fat during a lifestyle intervention in non-alcoholic fatty liver disease. Gut 2009; 58: 1281 - 1288

[19] Brouwers B, Hesselink MK, Schrauwen P et al. Effects of exercise training on intrahepatic lipid content in humans. Diabetologia 2016; 59: 2068-2079

[20] Slentz CA, Bateman LA, Willis LH et al. Effects of aerobic vs. resistance training on visceral and liver fat stores, liver enzymes, and insulin resistance by HOMA in overweight adults from STRRIDE AT/RT. Am J Physiol Endocrinol Metab 2011; 301: E1033-E1039

[21] Sarzynski MA, Burton J, Rankinen T et al. The effects of exercise on the lipoprotein subclass profile: A meta-analysis of 10 interventions. Atherosclerosis 2015; 243: 364-372

[22] Hayashino Y, Jackson JL, Fukumori $\mathrm{N}$ et al. Effects of supervised exercise on lipid profiles and blood pressure control in people with type 2 diabetes mellitus: a meta-analysis of randomized controlled trials. Diabetes Res Clin Pract 2012; 98: 349-360

[23] Interdisziplinäre Leitlinie der Qualität S3 zur „Prävention und Therapie der Adipositas“ 2014. AWMF-Register Nr. 050/001. Im Internet: http://www.awmf.org/leitlinien/detail/I/050001.html. Stand: 21.03.2017

[24] Niess A, Thiel A. Ursachen der Adipositas: Änderung des Bewegungsverhaltens. In: Bischoff SC, Hrsg. Adipositas -
Grundlagen und Praxis. Mechanismen, Prävention und Therapie. Berlin: Walter de Gruyter Verlag (im Druck)

[25] Donath MY. Multiple benefits of targeting inflammation in the treatment of type 2 diabetes. Diabetologia 2016; 59: $679-682$

[26] Karstoft K, Pedersen BK. Exercise and type 2 diabetes: focus on metabolism and inflammation. Immunol Cell Biol 2016; 94: $146-150$

[27] Handschin C, Spiegelman BM. The role of exercise and PGC1 $\alpha$ in inflammation and chronic disease. Nature 2008; 454: 463-469

[28] Hopps E, Canino B, Caimi G. Effects of exercise on inflammation markers in type 2 diabetic subjects. Acta Diabetol 2011; 48: $183-189$

[29] Hayashino Y, Jackson JL, Hirata T et al. Effects of exercise on C-reactive protein, inflammatory cytokine and adipokine in patients with type 2 diabetes: a meta-analysis of randomized controlled trials. Metabolism 2014; 63: 431 - 440

[30] Sixt S, Beer S, Blüher M et al. Long- but not short-term multifactorial intervention with focus on exercise training improves coronary endothelial dysfunction in diabetes mellitus type 2 and coronary artery disease. Eur Heart J 2010; 31: 112 - 119

[31] Ribeiro F, Alves AJ, Duarte JA et al. Is exercise training an effective therapy targeting endothelial dysfunction and vascular wall inflammation? Int J Cardiol 2010; 141: 214-221

[32] Olver TD, Laughlin MH. Endurance, interval sprint, and resistance exercise training: impact on microvascular dysfunction in type 2 diabetes. Am J Physiol Heart Circ Physiol 2016; 310: $\mathrm{H} 337-\mathrm{H} 350$

[33] Voulgari C, Pagoni S, Vinik A et al. Exercise improves cardiac autonomic function in obesity and diabetes. Metabolism 2013; 62: 609-621

[34] ADA. Foundations of cara: education, nutrition, physical activity, smoking cessation, psychological care, and immunization. Sec. 4. In Standards of Medical Care in Diabetes - 2015. Diabetes Care 2015; 38: S20 - S30

[35] Church TS, Cheng Y], Earnest CP et al. Exercise capacity and body composition as predictors of mortality among men with diabetes. Diabetes Care 2004; 27: 83 - 88

[36] Lee DC, Sui X, Artero EG et al. Long-term effects of changes in cardiorespiratory fitness and body mass index on all-cause and cardiovascular disease mortality in men: the Aerobics Center Longitudinal Study. Circulation 2011; 124: 2483 - 2490

[37] Sawada SS, Lee IM, Naito $\mathrm{H}$ et al. Long-term trends in cardiorespiratory fitness and the incidence of type 2 diabetes. Diabetes Care 2010; 33: 1353-1357

[38] Thamer C, Stumvoll M, Niess A et al. Reduced skeletal muscle oxygen uptake and reduced beta-cell function: two early abnormalities in normal glucose-tolerant offspring of patients with type 2 diabetes. Diabetes Care 2003; 26: 2126-2132

[39] Bouchard C, Rankinen T. Individual differences in response to regular physical activity. Med Sci Sports Exerc 2001; 33: S446-\$451

[40] Bouchard C, Blair SN, Church TS et al. Adverse metabolic response to regular exercise: is it a rare or common occurrence? PLoS One 2012; 7: e37887

[41] Böhm A, Weigert C, Staiger $\mathrm{H}$ et al. Exercise and diabetes: relevance and causes for response variability. Endocrine 2016; 51: $390-401$

[42] Pandey A, Swift DL, McGuire DK et al. Metabolic effects of exercise training among fitness-nonresponsive patients with type 2 diabetes: The HART-D Study. Diabetes Care 2015; 38: $1494-1501$ 
[43] Henson J, Davies M], Bodicoat DH et al. Breaking up prolonged sitting with standing or walking attenuates the postprandial metabolic response in postmenopausal women: a randomized acute study. Diabetes Care 2016; 39: 130 -138

[44] Bailey DP, Broom DR, Chrismas BC et al. Breaking up prolonged sitting time with walking does not affect appetite or gut hormone concentrations but does induce an energy deficit and suppresses postprandial glycaemia in sedentary adults. Appl Physiol Nutr Metab 2016; 41: 324-331

[45] Peddie MC, Bone JL, Rehrer NJ et al. Breaking prolonged sitting reduces postprandial glycemia in healthy, normal-weight adults: A randomized crossover trial. Am J Clin Nutr 2013; 98 : $358-366$

[46] Van Dijk JW, Venema M, Van Mechelen W et al. Effect of moderate-intensity exercise versus activities of daily living on 24-hour blood glucose homeostasis in male patients with type 2 diabetes. Diabetes Care 2013; 36: 3448 - 3453

[47] Sigal RJ, Armstrong CEP, Colby P et al. Clinical Practice Guidelines. Physical Activity and Diabetes. Can J Diabetes 2013; 37 : S40 - S44

[48] Pollock ML, Franklin BA, Balady G], AHA Science Advisory et al. Resistance exercise in individuals with and without cardiovascular disease: benefits, rationale, safety, and prescription: An advisory from the Committee on Exercise, Rehabilitation, and Prevention, Council on Clinical Cardiology, American Heart Association; Position paper endorsed by the American College of Sports Medicine. Circulation 2000; 101: 828-833

[49] ACSM Position Stand: The recommended quantity and quality of exercise for developing and maintaining cardiorespiratory and muscular fitness, and flexibility in healthy adults. Med Sci Sports Exerc 1998; 30: 975-991

[50] Weston KS, Wisløff U, Coombes JS. High-intensity interval training in patients with lifestyle-induced cardiometabolic disease: a systematic review and meta-analysis. $\mathrm{Br}$ J Sports Med 2014; 48: $1227-1234$

[51] Tjønna AE, Lee SJ, Rognmo $\emptyset$ et al. Aerobic interval training versus continuous moderate exercise as a treatment for the metabolic syndrome: a pilot study. Circulation 2008; 118 : $346-354$

[52] Adams ], Ogola G, Stafford P et al. High-intensity interval training for intermittent claudication in a vascular rehabilitation program. J Vasc Nurs 2006; 24: $46-49$

[53] Stefan N, Niess A. Körperliche Aktivität, Sport und Training. In: Häring HU, Gallwitz B, Müller-Wieland D, Usadel KH, Mehnert H, Hrsg. Diabetologie in Klinik und Praxis. Stuttgart: Thieme; 2011: $317-325$

[54] Unick JL, Gaussoin SA, Hill JO et al. Four-year physical activity levels among intervention participants with type 2 diabetes. Med Sci Sports Exerc 2016; 48: 2437 - 2445

[55] Carrell SE, Hoekstra M, West JE. Is poor fitness contagious?: Evidence from randomly assigned friends. Journal of Public Economics 2011; 95: 657-663

[56] Castellani W, Ianni L, Ricca V et al. Adherence to structured physical exercise in overweight and obese subjects: a review of psychological models. Eat Weight Disord 2003; 8: 1-11

[57] Delahanty LM, Conroy MB, Nathan DM, Diabetes Prevention Program Research Group. Psychological predictors of physical activity in the diabetes prevention program. J Am Diet Assoc 2006; 106: 698-705

[58] Plotnikoff RC, Lippke S, Trinh L et al. Protection motivation theory and the prediction of physical activity among adults with type 1 or type 2 diabetes in a large population sample. $\mathrm{Br}$ J Health Psychol 2010; 15: 643-661
[59] Robertson C, Archibald D, Avenell A et al. Systematic reviews of and integrated report on the quantitative, qualitative and economic evidence base for the management of obesity in men. Health Technol Assess 2014; 18: 1-424

[60] Korkiakangas EE, Alahuhta MA, Laitinen JH. Barriers to regular exercise among adults at high risk or diagnosed with type 2 diabetes: a systematic review. Health Promot Int 2009; 24 : 416-427

[61] Grace SL, Barry-Bianchi S, Stewart DE et al. Physical activity behavior, motivational readiness and self-efficacy among Ontarians with cardiovascular disease and diabetes. J Behav Med 2007; 30: $21-29$

[62] Sweet SN, Fortier MS, Guérin E et al. Understanding physical activity in adults with type 2 diabetes after completing an exercise intervention trial: A mediation model of self-efficacy and autonomous motivation. Psychol Health Med 2009; 14 : $419-429$

[63] Thiel A, Thedinga HK, Thomas SL et al. Have adults lost their sense of play? An observational study of the social dynamics of physical (in)activity in German and Hawaiian leisure settings. BMC Public Health 2016; 16: 689

[64] Bartlett JD, Close GL, MacLaren DP et al. High-intensity interval running is perceived to be more enjoyable than moderateintensity continuous exercise: implications for exercise adherence. J Sports Sci 2011; 29: 547 - 553

[65] Foster C, Farland CV, Guidotti F et al. The effects of high intensity interval training vs steady state training on aerobic and anaerobic capacity. J Sports Sci Med 2015; 14: 747 - 755

[66] Kirk A, Mutrie N, Maclntyre P et al. Increasing physical activity in people with type 2 diabetes. Diabetes Care 2003; 26: $1186-1192$

[67] Yoo M, D’Silva L], Martin K et al. Pilot study of exercise therapy on painful diabetic peripheral neuropathy. Pain Med 2015; 16 : $1482-1489$

[68] Pop-Busui R. What do we know and we do not know about cardiovascular autonomic neuropathy in diabetes. J Cardiovasc Transl Res 2012; 5: 463-478

[69] Kenny GP, Sigal RJ, McGinn R. Body temperature regulation in diabetes. Temperature (Austin) 2016; 3: 119-145

[70] Bax J], Young LH, Frye RL, ADA et al. Screening for coronary artery disease in patients with diabetes. Diabetes Care 2007; 30: $2729-2736$

[71] Deutsche Gesellschaft für Sportmedizin und Prävention (DGSP). S1-Leitlinie Vorsorgeuntersuchung im Sport. 2007. Im Internet: http://www.bayerischersportaerzteverband.de/ fileadmin/user_upload/html/Download/S1_Leitlinie_DGSP. pdf Stand: 21.03.2017

[72] Kemmer FW, Halle M, Stumvoll M et al. Diabetes Sport und Bewegung. DDG Praxisempfehlung. Diabetologie 2012; 7: $\mathrm{S} 170-\mathrm{S} 172$

[73] Gordon BA, Bird SR, Maclsaac RJ et al. Does a single bout of resistance or aerobic exercise after insulin dose reduction modulate glycaemic control in type 2 diabetes? A randomised cross-over trial. J Sci Med Sport 2016; 19: 795 - 799

\section{Bibliografie}

DOI http://dx.doi.org/10.1055/s-0042-121407 | Diabetologie 2017; 12: 112-126 @ Georg Thieme Verlag KG Stuttgart · New York ISSN 1861-9002 


\section{CME-Fragen bei CMIEthieme.de}

Viel Erfolg bei Ihrer CME-Teilnahme unter http://cme.thieme.de

Bitte informieren Sie sich über die genaue Gültigkeitsdauer unter http://cme.thieme.de

Sollten Sie Fragen zur Online-Teilnahme haben, unter http://cme.thieme.de/hilfe

finden Sie eine ausführliche Anleitung.

Unter https://eref.thieme.de/ZZWHN6W oder über den QR-Code kommen Sie direkt zum Artikel zur Eingabe der Antworten.

VNR 2760512017152373397

\section{Frage 1}

Welcher der folgenden Effekte ist im Rahmen eines regelmäßigen Trainings beim Typ-2-Diabetes nicht zu erwarten?
A Zunahme der körperlichen Fitness
B Verringerung ektoper Fettdepots
C Abnahme des HBA1c
D Rückbildung signifikanter Koronarstenosen
E Steigerung der Fettsäureoxidation

\section{Frage 2}

Welche Änderung des HBA1c ist im Mittel unter einem leitliniengerecht durchgeführten Ausdauertraining zu erwarten?
A Abnahme um $1,9 \%$ (z. B. von 8,9 auf $7,0 \%$ )
B Abnahme um $0,75 \%$
$C$ keine Beeinflussung
D Zunahme um $0,75 \%$
E Abnahme um $0,1 \%$

\section{Frage 3}

Welche Aussage zum Effekt körperlicher Aktivität auf das Übergewicht ist falsch? Zum Erreichen einer erfolgreichen Gewichtsreduktion ...

A sollten wöchentlich zumindest $1200-2000$ kcal zusätzlich durch körperliche Aktivität verbrannt werden.

$B$ bietet ein Krafttraining Vorteile gegenüber einem Ausdauertraining.

C ist es nicht notwendig, ausschließlich im niedrigen Intensitätsbereich (unter $50 \%$ der maximalen Herzfrequenz) zu belasten.

D können unterschiedliche Formen des Ausdauertrainings (Radfahren, Walking, Laufen, Schwimmen) genutzt werden.

E sollte bei Neueinsteigern der Trainingsumfang langsam gesteigert werden.

\section{Frage 4}

Welche Empfehlung zur körperlichen Aktivität bei Typ-2-Diabetes ist in den Leitlinien nicht aufgeführt?

A wöchentlich mindestens 150 min moderat-intensives Ausdauertraining

B wöchentlich mindestens 2 Trainingseinheiten Krafttraining

C Verteilung des Ausdauertrainings auf mindestens 3 Trainingstage pro Woche

D Inaktivitätsphasen wie Sitzen sollten alle 90 min unterbrochen werden

E Meiden eines intensiven Intervalltrainings

\section{Frage 5}

In welcher Situation bzw. bei welchem Folgeschaden ist bei Patienten mit Typ-2-Diabetes ein intensives Ausdauertraining zunächst kontraindiziert?

A leichtgradige nicht proliferative Retinopathie

B periphere Neuropathie

C Albuminurie

D Vorliegen einer Ketose

E Blutzucker von $301 \mathrm{mg} / \mathrm{dl}$ ohne Ketosenachweis

\section{Frage 6}

Welche Maßnahme muss bei einem mit Insulin behandelten Patienten mit Typ-2-Diabetes zur Vorbeugung einer Hypoglykämie beim körperlichen Training in der Regel nicht empfohlen werden?

A Verzicht auf ein intensives Ausdauertraining

B Zufuhr von 15-30 g Kohlenhydrate bei einem Blutzucker unter $100 \mathrm{mg} / \mathrm{dl}$ vor Trainingsbeginn

C Reduktion der Insulindosierung vor dem Training

D häufigere Messung des Blutzuckers

E Mitführen von Extra-BE beim Training

- Weitere Fragen auf der folgenden Seite... 


\section{CME-Fragen bei CME.thieme.de}

Fortsetzung $\ldots$

\section{Frage 7}

Welche Aussage zur körperlichen Fitness bei Typ-2-Diabetes ist richtig?

A Sie ist bei Patienten mit Typ-2-Diabetes fast immer eingeschränkt.

B Sie kann durch ein körperliches Training in der Regel nur geringgradig gesteigert werden.

C Sie zeigt bei Patienten mit Typ-2-Diabetes eine negative Assoziation zum Sterblichkeitsrisiko.

D Ohne ihre gleichzeitige Zunahme führt ein körperliches Training nicht zu einer Verbesserung der glykämischen Kontrolle.

E Sie kann nur dann gesteigert werden, wenn ein kombiniertes Training aus Kraft- und Ausdauertraining durchgeführt wird.

\section{Frage 8}

In welchem Intensitätsbereich in Bezug (\%) auf die maximale Sauerstoffaufnahme soll bei Typ-2-Diabetes der Trainingsreiz in einem aeroben Ausdauertraining nach der Dauermethode gesetzt werden?
A $20-30 \%$
B $10-40 \%$
C $40-85 \%$
D $80-90 \%$
E unter $50 \%$

\section{Frage 9}

In welchem Intensitätsbereich in Bezug (\%) auf die Maximalkraft (1-RM, One Repetition Maximum) soll bei Typ-2-Diabetes der Trainingsreiz in einem Krafttraining gesetzt werden, wenn ein erster Trainingsaufbau bereits erfolgt ist?
A $75-80 \%$
B unter $40 \%$
C $40-50 \%$
D über $90 \%$
E bei $100 \%$

\section{Frage 10}

Welche Diagnose kann die körperliche Belastbarkeit bei einem Patienten mit Typ-2-Diabetes so einschränken, dass eine absolute Kontraindikation für ein Krafttraining besteht?

\section{A Gonarthrose}

B arterielle Hypertonie Grad I

C Z.n. Stentimplantation in die LAD (Ramus interventricularis anterior)

D chronische Herzinsuffizienz NYHA II

E pro>liferative Retinopathie 